\title{
Underlying dimensions in the perceptual space of perfumery odors
}

\author{
Manuel Zarzo Castello \\ From 1st International Workshop on Odor Spaces \\ Hannover, Germany. 4-7 September 2013
}

The perceptual space of odors is highly multidimensional, and consequently it is difficult to reach a consensus about how to develop two-dimensional (2D) sensory odor maps. The problem is simplified if we focus on a subset of smells (e.g., odors of wine, beer, cheese, etc.), and many sensory maps have been proposed for such perceptual subspaces. Perfumes cover a wide spectrum of odor qualities, but they are obviously biased towards pleasant non-food scents. Various sensory maps have been proposed in the literature for those odor descriptors most frequently applied in perfumery, but no standard exists yet.

The multivariate statistical analysis of odor profile databases provides valuable information to better understand the underlying dimensions of olfactory perception space. The analysis of two odor databases, compiled in the context of perfumery, is presented. The first one contains numeric odor profiles of 309 compounds based on 30 descriptors. The loading plot corresponding to the first and second principal components can be regarded as a sensory map of odor descriptors. Similar results were obtained in the analysis of another database, which comprises 66 descriptors and contains the semantic descriptions of 119 perfume materials. Results were compared with a 2D fragrance mapping reported in the literature, with Jellinek's Odor Effects Diagram as well as with Edwards' Fragrance Wheel, which classifies commercial perfumes into 14 categories. A striking consistency was observed in all cases [1], which indicates that it is possible to develop standard 2D sensory maps of perfumery odor descriptors if a consensus is first reached regarding which odorants best represent particular odor qualities.

One underlying dimension discriminates scents as feminine versus masculine. The other one differentiates cool/refreshing vs. warm odors, which might reflect a perceptual construct related with temperature. It is well known by perfumers that scents perceived as 'cooling' and 'refreshing' (e.g. citrus and green notes) tend to evaporate quickly. Conversely, those described as 'warm' (e.g., oriental, powdery, erogenic and animalic scents) are tenacious. Tenacity or substantivity is the parameter that measures the lasting property of a material when applied on the skin. In a recent study, perceptual olfactory ratings on a 'fresh' scale (i.e., cool vs. warm) were compared with substantivity parameters from the literature, and a tight correlation $(r=-0.85)$ was obtained [2].

Assuming that odor perception space was shaped throughout evolution in temperate climates, results reported here are consistent with the hypothesis that 'cool/fresh' evokes scents typically encountered in the cool season, while 'warm' would be evoked by odors found in nature during summer. This hypothesis might reflect the role of olfaction in chronobiological rhythms and may provide a new insight to better understand the perceptual space of perfumery scents.

Published: 16 April 2014

\section{References}

1. Zarzo M, Stanton DT: Understanding the underlying dimensions in perfumers' odor perception space as a basis for developing meaningful odor maps. Atten Percept Psychophys 2009, 71:225-47.

2. Zarzo M: What is a Fresh Scent in Perfumery? Perceptual Freshness is Correlated with Substantivity. Sensors (Basel) 2012, 13:463-83.

doi:10.1186/2044-7248-3-S1-020

Cite this article as: Castello: Underlying dimensions in the perceptual space of perfumery odors. Flavour 2014 3(Suppl 1):O20. 